\title{
Mfa5 from Porphyromonas gingivalis: a von Willebrand factor domain and an intramolecular isopeptide bond in a Gram-negative bacterial fimbrial protein
}

\section{Thomas Heidler}

Umea University https://orcid.org/0000-0002-5997-3945

\section{Rolf Claesson}

Department of Odontology

Karina Persson ( $\nabla$ karina.persson@umu.se )

Ume\&\#x00E5; University https://orcid.org/0000-0003-0807-0348

\section{Article}

Keywords:

Posted Date: July 7th, 2020

DOI: https://doi.org/10.21203/rs.3.rs-38404/v1

License: (9) This work is licensed under a Creative Commons Attribution 4.0 International License.

Read Full License 


\section{Abstract}

The Gram-negative bacterium Porphyromonas gingivalis is a secondary colonizer of the oral biofilm and is involved in the onset and progression of periodontitis. Its fimbriae, of type- $\mathrm{V}$, are important for attachment to other microorganisms in the biofilm and for adhesion to host cells. The fimbriae are assembled from five proteins encoded by the mfa1 operon, of which Mfa5 is one of the ancillary tip proteins. Here we report the X-ray structure of the N-terminal half of Mfa5, which reveals a von Willebrand factor domain and two IgG-like domains. One of the IgG-like domains is stabilized by an intramolecular isopeptide bond, which is the first such bond observed in a Gram-negative bacterium. These features make Mfa5 structurally more related to streptococcal adhesins than to the other P. gingivalis Mfa proteins. The structure reported here indicates that horizontal gene transfer has occurred among the bacteria that within the oral biofilm.

\section{Introduction}

Fimbriae (also called pili) are long filamentous protein polymers that project from the bacterial surface and are crucial for attachment to other microorganisms, host cells, and surfaces [1]. They usually contain several protein subunits encoded by the same gene cluster, resulting in the assembly of a long shaft of repetitive proteins decorated by ancillary tip proteins [2-4]. For some gene clusters, flanking transposons have been identified indicating that horizontal gene transfer can occur [5]. In Gram- negative bacteria, these filamentous structures are assembled in a non-covalent manner assisted by multi-protein complexes that span either the outer membrane or both the outer and inner membranes. In contrast, Gram-positive fimbrial proteins are covalently linked to each other by intermolecular isopeptide bonds, which are amide bonds between a lysine side chain of one subunit and the carboxyl group of a C-terminal threonine of the next subunit. The formation of these bonds is mediated by specific sortases that are encoded by the same gene cluster [6,7]. To date six different types of fimbriae have been classified: 1 ) type-I, also called the chaperone-usher type, 2) type-IV fimbriae, 3) type-IV secretion fimbriae, 4) type-V fimbriae, 5) curli fibers, and 6) sortase-mediated fimbriae, all of which are essential for bacterial virulence $[2,4]$.

Porphyromonas gingivalis is a Gram-negative, rod shaped, anaerobic bacteria that belongs to the bacteroidetes phylum [8]. P. gingivalis colonizes the oral biofilm as a secondary colonizer using primary colonizers, mainly commensal streptococci, as attachment partners $[9,10]$. The proliferation of $P$. gingivalis can lead to a shift from a healthy biofilm composed mainly of commensals to a more pathogenic biofilm [8]. P. gingivalis causes chronic inflammation (periodontitis) [11-13] and is believed to be involved in the onset of systemic diseases such as various cancers, rheumatoid arthritis, heart diseases, diabetes, pregnancy complications, and Alzheimer's disease [14-20].

P. gingivalis expresses two antigenically distinct but structurally homologous type- $V$ fimbriae that are important both for attachment to other bacteria and for invasion of different host tissues [9, 21-25]. FimA fimbriae are up to $2 \mu \mathrm{m}$ long and Mfa1-fimbriae are $100 \mathrm{~nm}$ long $[26,27]$, and both have been 
shown to be involved in disease progression [28]. The $m f a 1$ and fimA gene clusters encode five proteins each, including one major stalk subunit (Mfa1 or FimA), a cell-wall anchor (Mfa2 or FimB), and three ancillary tip proteins (Mfa3-5 or FimC-E) [29]. The individual proteins harbor an N-terminal signal peptide that leads them to be transported into the periplasm via the Sec pathway [30]. Within the periplasmic space, the proteins are acylated, processed by signal peptidase II, and transported to the outer membrane by the localization of lipoprotein export pathway [31].

Bacteria of the bacteroidetes phylum have a specific secretion system, type-IX, that transports certain proteins across the outer membrane [32], and proteins translocated by this system are selected by a conserved C-terminal signal domain. Among the fimbrial proteins such a C-terminal signal domain is only found in Mfa5. Blocking the type-IX secretion system in P. gingivalis inhibits export of Mfa5 to the outer membrane, whereas the other fimbrial proteins are not affected [33], indicating that they are transported over the membrane using another, unknown pathway. Mfa5 is also unique among the fimbrial proteins in that it is exceptionally large (1,228 amino acids), and whereas the Mfa $1-4$ proteins consist of two $\beta$ sandwich domains, Mfa5 is predicted to contain several domains, one of which is a von Willebrand factor (vWF) domain.

In this study we present the crystal structure of the N-terminal half of Mfa5, residues $99-664$, at $1.8 \AA$ resolution. Our high-resolution structure reveals unforeseen similarity to adhesins hitherto only observed in Gram-positive bacteria. As predicted, the structure contains a vWF domain with a complete metal iondependent adhesion site (MIDAS) as well as two IgG-like domains. Intriguingly, one of the IgG-like domains is stabilized by an intramolecular isopeptide bond, which to our knowledge is the first such bond observed in a Gram-negative bacterial surface protein.

\section{Results}

\section{Structure determination of Mfa5}

A construct lacking the $\mathrm{N}$ - and $\mathrm{C}$-terminal signal peptides, Mfa521-1044, was designed and expressed in E. coli (Fig. 1). The calculated molecular weight was $112 \mathrm{kDa}$, however purification resulted in a degradation product estimated to $\sim 70 \mathrm{kDa}$ by SDS-PAGE. A similar fragment was obtained when the

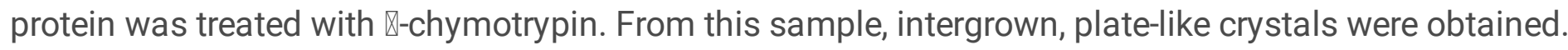
The crystals diffracted to $1.8 \AA$, belonged to space group P212121, and contained one molecule in the asymmetric unit. However, no phase information could be obtained so a shorter construct, Mfa5138-435, encompassing only the predicted vWF domain was crystallized. One single rectangular crystal diffracting to $1.85 \AA$ in space group P21 and with one molecule in the asymmetric unit was obtained. A highly redundant dataset was collected, and the structure was solved using sulfur SAD. The structure was refined to final Rwork and Rfree of 13 and $18 \%$. Next, this model was used to solve the structure of the 70 $\mathrm{kDa}$ fragment by molecular replacement. A model encompassing amino acids 98 to 664 could be built into the density and successively refined to Rwork and Rfree of 16 and $20 \%$. Final refinement statistics for both structures are presented in Table 1. 
From the crystallized $70 \mathrm{kDa}$ fragment a model that spans residues $99-664$ could be built. The structure consists of three domains (D1 to D3) that build up an elongated shape with dimensions of $44 \AA \times 48 \AA \times$ $164 \AA$ (Fig. 1). This model will be referred to as MfaD1-D3. The first domain, D1, (amino acids 142-418), forms a Rossman fold classified as a vWF domain (Fig. 2 \& 3a) [34]. The vWF domain comprises a cleft harboring a MIDAS motif with a bound $\mathrm{Mg}^{2+}$ ion. In addition, a $\mathrm{Ca}^{2+}$ ion is coordinated by an adjacent loop region (amino acids 298-303).

The second domain, D2 (amino acids 105-135 and 423-544), has an IgG-like fold composed of a $\beta$ sandwich with three and five $\beta$-strands, respectively. Intriguingly, the vWF domain is inserted between strands $B$ (first sheet) and C (second sheet) of the D2 domain. The overall topology of D2 is similar to CnaA, which is one of the common building blocks of Gram-positive adhesins [35]. Interestingly, an intramolecular isopeptide bond connects Lys111 of the first $\beta$-sheet to Asn518 of the second $\beta$-sheet (Fig. $2 \& 3 b)$.

D3, encompassing amino acids 547-664, comprises six $\beta$-strands in a twisted tube-like $\beta$-sheet that can be classified as a ubiquitin-like roll. A topology plot shows that there is a similarity to the lgG-like CnaB fold of Gram-positive bacterial adhesins (Fig. 2 \& 3c) [35]; however, the generally high B-factor and comparisons to other IgG-like domains led to the assumption that D3 might be incomplete.

von Willebrand factor domain

The structure obtained from Mfa5138-435, that will be referred to as Mfa5D1, is folded into a vWF domain consisting of a typical Rossman fold comprising a six-stranded $\beta$-sheet surrounded by three helices on each side. This globular domain, which has a wrench-like form, has the dimensions $44 \AA \times 52$ $\AA \times 79 \AA$ and includes the MIDAS motif, which is a central feature for interaction with various targets. In the Mfa5 MIDAS site, the five residues coordinating the metal ion are situated on the wrench head on top of the $\beta$-sheet. Mfa5 has a classical MIDAS motif [36] in which the side chain oxygens of Ser152, Ser154, and Thr224 coordinate the metal directly and the side chains of Asp150 and Asp252 coordinate the metal via a water molecule. The crystallization conditions and the coordination distances suggest that this is a $\mathrm{Mg}^{2+}$ ion (bottom insert in Fig. 2). A homology search with DALI [37] using both Mfa5D1 and Mfa5D1-D3 indicated a close structural relationship to the Gram-positive tip pilins RrgA (PDBID: 2ww8 [38]) and GBS104 (PDBID: 3txa [39]) from Streptococcus pneumoniae and Streptococcus agalactiae as well as to human integrins such as the collagen-binding a2-I domain (PDBID: 1dzi [40]). A superposition of their respective vWF domains to the VWF domain of Mfa5 gives a good match to the streptococcal tip fimbriae (rmsd $1.9 \AA$ (151 of 296 Ca-positions)) and the a2-I vWF domain (rmsd $2.5 \AA$ (88 of 296 Ca-positions)). This was unexpected based on their low sequence similarity and identity to Mfa5, $19 / 10 \%$ for the streptococcal adhesins and $21 / 9 \%$ for the a2-I protein. A structure-based sequence alignment of the vWF domain confirmed that the MIDAS site is conserved (Fig. S1). 
Compared to the a2-I structure, the bacterial vWF domains proteins have several additional structural elements. Similar to GBS104 and RrgA [38, 39], the Mfa5 vWF domain has a long insertion (ARM2) between the fourth $\beta$-strand and the fourth a-helix of the domain (amino acids 257-355). In Mfa5, ARM2 folds into a small domain comprised of long loops centered around a $\beta$-sheet of four very short strands. Two helices, separated by a proline, form an L-shaped structure that covers one side of the sheet. One of the loops in the ARM2 domain coordinates the second metal in the structure. The metal is coordinated by the main chain carbonyls of Asn298, Thr301, and Leu303 and by the side chain oxygens of Asn298 and Thr301. In addition, the side chain of Asp336 from another loop also coordinates the metal. The conformation of the loop is additionally constrained by the three prolines Pro299, Pro302, and Pro305. Based on the coordination distances, this metal has been modeled as a calcium ion. Interestingly, the whole ARM2 domain is rich in prolines, 13 of 98 residues, which contributes to the rigidity of the loops (Fig S2). The equivalent ARM2 in RrgA and GBS104 is longer, 123 amino acids (Fig. 4), and does not bind any metals. RrgA and GBS104 also have two additional loops, a 38 amino acid insertion within the $\beta$ strand C (ARM1) and a shorter loop on top of a2 (9 amino acids). These two insertions have no equivalents in Mfa5. Nonetheless, in all three bacterial proteins additional loops extend out from the vWF domain and create a lid over the top of the cleft harboring< the MIDAS site. Superposing integrin structures bound to extra cellular matrix proteins onto Mfa5 shows that the ARM2 domain of Mfa5, in its present conformation, would block the collagen binding shown for the integrin a2-I domain (PDBID: 1dzi [40]). In contrast, the ARM2 domain seems to mimic parts of the aV integrin domain, which together with $\beta 3$ integrin forms a cleft that binds to fibronectin (PDBID: 4mmx [41] (Fig. S3).

\section{The isopeptide bond stabilizes the D2 domain}

Although P. gingivalis is Gram-negative, the Mfa5 D2 domain has a fold that is common in surface molecules of Gram-positive bacteria. These domains in Gram-positive bacteria are often stabilized by intramolecular isopeptide bonds that are formed spontaneously between the side chains of a lysine and an asparagine or aspartate. Generally, these isopeptide bonds are formed in hydrophobic environments and need an adjacent acid to coordinate the participating side chains of the bond. Surprisingly, in the D2 domain of Mfa5 continuous electron density from the Lys111 $\varepsilon$-amino group to the $\delta$-carboxyamide group of Asn518 is observed, which clearly indicates an intramolecular isopeptide bond (top inlay in Fig. 2). The surrounding environment is mainly hydrophobic, and the only acid close to the isopeptide bond is Asp432, which is not at hydrogen bonding distance (3.7 and $4.0 \AA$ to the nitrogen and oxygen of the bond, respectively). Instead, the isopeptide bond is stabilized by Thr517 (OG) and by Tyr486 (OH) via a water molecule. An identical arrangement, with an isopeptide bond formed by Lys and Asn residues with coordinating residues Asp, Thr, and Tyr are found in the D2 and N2 domains of RrgA and GBS104, respectively (Fig. S2). The intramolecular isopeptide bond in Mfa5 links the two $\beta$-sheets of the lgG-like domain; Lys111 is located on the first $\beta$-strand of the first $\beta$-sheet and Asn518 is located on an antiparallel $\beta$-strand of the second $\beta$-sheet. 
To further validate the presence of the isopeptide bond, purified Mfa599-664 and its point mutant K111A were used in ESI-TOF mass spectrometry. This method measures the molecular mass with an accuracy of $\pm 1 \mathrm{Da}$, and it gave a mass for Mfa599-664 of $62,043 \mathrm{Da}$, which was $15.95 \mathrm{Da}$ less than the theoretical molecular mass of 62,059 Da. This difference in mass indicates the loss of one NH3 group (17 Da), thus verifying the formation of an isopeptide bond (Fig. S4, S5, S6). In contrast, the measured mass of 62,002

Da for the Mfa599-664 K111A mutant was identical to the theoretical molecular mass. To further analyze if this intramolecular isopeptide bond has an influence on protein stability, a thermal shift assay was performed on both proteins. The Mfa599-664 protein showed a two-step unfolding pattern with $\mathrm{T} m$ s at $66^{\circ} \mathrm{C}$ and $78^{\circ} \mathrm{C}$, indicating two domains. In the $\mathrm{K} 111 \mathrm{~A}$ mutant, a shoulder at $66^{\circ} \mathrm{C}$ remained whereas the second peak shifted to $71^{\circ} \mathrm{C}$ (Fig. 5). The resulting $7^{\circ} \mathrm{C} \mathrm{T} m$ difference for the second peak and the overall difference in unfolding pattern indicate that the absence of the isopeptide bond has a destabilizing effect on the protein.

\section{Mfa5 integration in the fimbriae}

The $m f a$ gene cluster encodes five proteins, of which Mfa5 has been confirmed as a substrate for the type-IX secretion system [33]. Because native Mfa5 fimbriae from $P$. gingivalis contain Mfa1, 3, 4, and 5 as previously described [42], we decided to derive specific antibodies against Mfa5 and one additional tip protein, Mfa3. When analyzing native fimbriae by SDS-PAGE, the bands at 120-150 kDa and $40 \mathrm{kDa}$ were subsequently verified by western blotting to belong to Mfa5 and Mfa3, respectively (Fig. 6). Using the same Mfa5 antibody serum and pure native fimbriae for negative staining, a clear elongation of the fimbriae was detectable (Fig. 7). Bulky connectors roughly in the middle of two 100 nm long fimbriae indicate an antibody connecting two fimbriae head to head (white arrows).

\section{Discussion}

Fimbriae are protein polymers projecting from the bacterial surface, and these make the first contact with the targeted host. Therefore, it is of great interest to understand the structure, assembly mechanism, and ligand specificity of fimbriae because their adherence mechanisms and biogenesis are potential targets for the development of novel targeted antibacterials [43]. In recent decades, the combined efforts of many groups have contributed to the understanding of both the ligand specificity and the donor-strand exchange mechanism that underlie the polymerization of type-I fimbriae in Gram-negative bacteria such as $E$. coli (phylum Proteobacteria). This has led to the development of novel antibacterial treatments that have gone as far as clinical trials [4]. P. gingivalis, on the other hand, belongs to the Bacteroidetes phylum and accordingly its two fimbriae, Mfa1 and FimA, are of type-V. Recent publications describing the X-ray structures of recombinant proteins building up type- $V$ fimbriae describe a common core structure of two domains, both comprising a $\beta$-sandwich [44-46] where the first strand of the protein is removed by gingipain proteases when the fimbriae are polymerized. These structures led to the suggestion that type- $V$ fimbriae also depend on a donor- strand exchange mechanism for bioassembly; however, the exact 
nature of this mechanism is still not fully understood [47]. The $m f a$ gene cluster encodes five proteins where the first four all have the classical type-V fold. The fifth protein, Mfa5, is different from the other Mfa proteins in many ways. It is considerably larger (1,228 residues) than Mfa1-4 (324-663 residues), and contrary to Mfa1, 3, and 4 it does not appear to be dependent on gingipains for maturation [33]. Instead, Mfa5 is the only fimbrial protein that has been found to be dependent on the type-IX secretion system for translocation across the outer membrane. Indeed, the crystal structures of the three N-terminal domains of Mfa5 that we present here do not resemble the other Mfa proteins. The three domains include one vWF domain (D1) and two IgG-like domains (D2 and D3). Surprisingly, despite being unrelated in sequence Mfa5 shows a striking similarity to the Gram-positive adhesins RrgA and GBS104, which both have a vWF domain and a number of IgG-like domains that appear to function as the stalk to project the VWF domain towards ligands, presumably those presented on host cells. The first Mfa5 IgG-like domain, D2, is formed from two segments of the protein, residues 105-138 that form the first two $\beta$-strands and residues 422-546 that constitute the rest of the domain. An intramolecular isopeptide bond between Lys111 and Asn518 links the first segment with the second. Keeping in mind that the Mfa5 D1, D2, and D3 domains presented here only comprise half of the full-length protein, it is possible to imagine that the full-length stalk might be constructed from several additional IgG-like domains. The D3 domain folds like an incomplete lgG-like domain, and comparison to the lgG-like domains in RrgA and GBS104 lead us to speculate that strands are missing from this domain. Hypothetically, extra strands might have been donated by a putative D4 domain if the protein had not been crystallized in a degraded form. Mfa5 constitutes the tip of the fimbria together with Mfa3 and Mfa4, and the association of these proteins in the fimbrial spatial arrangement is likely to have a stabilizing effect on the large Mfa5 protein compared to when it is expressed recombinantly on its own. The vWF domain is folded from 276 residues located between the two segments of the D2 domain. VWF domains are most commonly expressed by eukaryotic organisms but have also been found in some bacteria. In the human integrin a2-I, the vWF domain has its collagen-binding MIDAS motif exposed at the top of the protein, whereas bacterial vWF domains have extra segments (ARM1 and ARM2) folding as subdomains on the sides of the MIDAS motif. The streptococcal adhesins RrgA and GBS104 have two ARMs, whereas Mfa5 only has one. Superposition of the vWF domain of Mfa5 onto the human a2-I domain indicates that ARM2, in the present conformation, would interfere with collagen binding. Superposition of the vWF domain of Mfa5 onto integrin aVB3 indicates a closer mimic to its fibronectin binding cleft. Thus the VWF domain is a clear indication that the D1 domain of Mfa5 functions as an adhesin, but the ligand has yet not been determined.

The Mfa5 structure suggests that the ancestor of the $\mathrm{mfa} 5$ gene has been obtained from a streptoccocal ancestor. It has been shown that horizontal gene transfer between microorganisms happens frequently in the oral biofilm where hundreds of species live in close physical contact [48]. Over the course of evolution, Mfa5 has retained crucial structural features from its Gram-positive ancestor, such as the stabilizing intramolecular isopeptide bond and the conserved MIDAS motif, and has adapted to its Gram-negative host by adding a C-terminal domain that targets the protein to the type-IX secretion system, which is unique to the Bacteroidetes phylum. While the assembly mechanism of type- $\mathrm{V}$ fimbriae is not fully understood, it is known that the fimbrial proteins, that build up the final fimbria, are expressed as lipidated 
precursors that are transported to the outer membrane via the lipoprotein export system, [31] followed by gingipain-dependent removal of the first $\beta$-strand. This cleaved fimbrial protein can then polymerize through a donor-strand replacement mechanism in which a segment from the next fimbrial protein is predicted to participate [44-46]. Integration of Mfa5 into the fimbriae is not expected to follow this mechanism because Mfa5 is not dependent on the presence of gingipains; however, it has been indicated by in vivo studies that the incorporation of Mfa5 into the mature fimbriae requires a functional vWF domain [33]. This needs to be studied further because the truncation of the vWF domain might obstruct the correct formation of the other domains and thus its ability to bind to other fimbrial proteins.

Native fimbrial purifications from $P$. gingivalis, as analyzed by SDS gels, revealed protein bands that were identified as Mfa5 by Hasegawa et al. [42]. In this study we could further confirm that antibodies derived from recombinant Mfa5 specifically stain these bands. In negative staining images, the antibody against

Mfa5 appears to bind two fimbriae together, leading to the assumption that Mfa5 is indeed located at the tip of the fimbriae. The different conformations visible in the images also indicate high flexibility, which might be related to the linker regions between the domains. A structure of the full-length native fimbria would answer the remaining questions about the localization of the different subunits and their biogenesis.

$P$. gingivalis is a key pathogen and is strongly associated with the periodontitis that affects a large part of the population worldwide. The presence of $P$. gingivalis is believed to contribute to the onset of other systemic diseases such as Alzheimer's disease, rheumatoid arthritis, and oral and pancreatic cancer, and its capacity to bind early colonizers of the oral biofilm and to extracellular matrix proteins on host cells is deemed to be an important factor. Therefore, further development of anti-adhesive substances to block the vWF-binding cleft or the development of blocking antibodies will enable future tools to hinder the establishment and proliferation of this pathogen. Hindering the fimbriae from attaching to the primary colonizers of the oral biofilm is crucial because if $P$. gingivalis cannot colonize the mouth it will never cause the chronic inflammation that burdens the immune system and it will not have the opportunity to spread to other, non-oral parts of the body.

\section{Methods}

\section{Expression and purification of recombinant Mfa5 protein}

The primary Mfa5 sequence (GenBank AUR48966.1) was analyzed for conserved features using the webbased SIGNALP, PROSITE, INTERPRO and Conserved Domains Database, GOR4, SCRATCH, PSIPRED, and PHYRE2 tools [49-56]. Based on the combined results from all analyses, the full length Mfa5 was amplified by polymerase chain reaction from the $P$. gingivalis ATCC 33277 genome. Additional constructs, containing amino acids 21-1044, 138-435, 99-664, and 99-664 (K111A) were subsequently cloned by polymerase incomplete primer extension [57] into a pET-ZZ-1a [58] vector with flanking Ncol/Xhol sites (primers are presented in Table S1). 
The recombinant proteins were expressed in E. coli C41 (DE3) (Mfa521-1044) or BL21 (DE3 pLysS) (other constructs). Cells were cultivated at $20^{\circ} \mathrm{C}$ in $2-4 \mathrm{~L}$ Luria Bertani media supplemented with 50 $\mu \mathrm{g} \cdot \mathrm{mL}^{-1}$ kanamycin. At an OD600 of 0.8 the induction was started by the addition of $0.25 \mathrm{mM}$ IPTG. Cells were harvested $16 \mathrm{~h}$ later by centrifugation for $20 \mathrm{~min}$ at $4000 \times \mathrm{g}$, flash frozen in liquid nitrogen, and stored at $-80^{\circ} \mathrm{C}$ until further processing.

Cell pellets were resuspended in $50 \mathrm{~mL}$ cold phosphate-buffered saline (PBS) containing $260 \mathrm{mM} \mathrm{NaCl}$, $10 \mathrm{mM}$ imidazole, $2 \mathrm{mM} \beta$-mercaptoethanol, 1\% Triton X-100, and protease inhibitors (Pierce) and lysed by sonication. Cell debris was removed by centrifugation at $64,000 \times g$ for 30 min, and the supernatant was applied to Ni-IDA resin (TaKaRa Bio). After two wash steps, the protein was eluted with PBS and 250 $\mathrm{mM}$ imidazole. Tobacco Etch Virus protease or a-chymotrypsin at a 1:100 (w/w) ratio was added to the eluted sample, and the mixture was dialyzed against PBS overnight at $4^{\circ} \mathrm{C}$. Next, the sample was applied to a fresh Ni-resin column for re-chromatography. The cleaved protein was concentrated with an Amicon Ultra centrifugal filter (10 kDa cutoff; Millipore) and run on a Superdex200 16/60 column (GE Healthcare) in $20 \mathrm{mM}$ Tris $\mathrm{pH} 7.4$ and $100 \mathrm{mM} \mathrm{NaCl}$. Peak fractions were pooled, concentrated, and stored at $-80^{\circ} \mathrm{C}$ until further use.

\section{Cultivation of $P$. gingivalis}

Colonies of the $P$. gingivalis strains JI-1 fimA-deletion mutant ( $\triangle$ fimA) and $m f$ - 1 -deletion mutant $(\Delta$ mfa1),, both derived from $P$. gingivalis strain ATCC 33277, growing on blood agar medium were used to inoculate tryptic soy broth (TSB) supplemented with $2.5 \mathrm{~g} \cdot \mathrm{L}^{-1}$ yeast extract, $5 \mathrm{mg} \cdot \mathrm{L}^{-1}$ menadione, 2.5 $\mathrm{mg} \cdot \mathrm{L}^{-1}$ hemin, and $0.01 \mathrm{mg} \cdot \mathrm{L}^{-1}$ DTT overnight as previously described [9]. The cultures were preincubated at $37^{\circ} \mathrm{C}$ in an anaerobic chamber with an atmosphere of $85 \% \mathrm{~N} 2,5 \% \mathrm{CO}$, and $10 \% \mathrm{H} 2$. Aliquots of $250 \mathrm{~mL}$ of anaerobic TSB were subsequently inoculated with $5 \mathrm{~mL}(2 \%)$ overnight cultures of the two $P$. gingivalis mutants for $16-18 \mathrm{~h}$ and further processed.

\section{Purification of intact fimbriae}

A total of $1 \mathrm{~L}$ of $P$. gingivalis culture was harvested by centrifugation at $4,000 \times g$ for $20 \mathrm{~min}$. The cells were resuspended in $30 \mathrm{~mL} 20 \mathrm{mM}$ Tris pH 7.4, $10 \mathrm{mM} \mathrm{MgCl}$, $1.5 \mathrm{M} \mathrm{NaCl}, 10 \%$ sucrose, $0.1 \mathrm{mM}$ DTT, and DNase and lysed by four passes through a French press at 900 psi. Cell debris was separated by centrifugation at 9,000 x $g$ for $10 \mathrm{~min}$, and the supernatant was cleared at 143,000 $\mathrm{g}$ for $90 \mathrm{~min}$. The supernatant was saturated with $50 \% \mathrm{NH} 4 \mathrm{SO} 4$ at $4^{\circ} \mathrm{C}$, and a follow-up centrifugation at $15,000 \times g$ for 30 min was used to collect the precipitated protein. The pellet was resuspended and dialyzed overnight in 20 mM Tris pH 7.4 and 20 mM NaCl. A 1:2 diluted sample was applied on a HiTrap Q1 column (GE Healthcare) and eluted in a step gradient with $20 \mathrm{mM}$ Tris $\mathrm{pH} 7.4$ and $1 \mathrm{M} \mathrm{NaCl}$. Protein-containing fractions were pooled and applied on a HiPrep Sephacryl S-400 HR 16/60 column (GE Healthcare). Fractions corresponding to the peak were collected and applied to a Superose 6 Increase 10/300 column (GE Healthcare). Peak fractions were collected, and aliquots were flash frozen in liquid nitrogen for storage at $-80^{\circ} \mathrm{C}$. 


\section{Western blotting}

Mfa proteins were detected in fimbrial purifications by western blotting. An SDS gel was loaded with 50 ng detectable protein per lane. After electrophoresis, the gel was incubated together with blotting paper and membrane ( $0.45 \mu \mathrm{m}$ nitrocellulose, GE Healthcare) in Towbin buffer $(25 \mathrm{mM}$ Tris pH 8.3, $192 \mathrm{mM}$ glycine, $20 \%(\mathrm{v} / \mathrm{v})$ methanol) for 5-10 min. Next, the blot was assembled in a semi-dry blotter and run at $15 \mathrm{~V}$ for $15 \mathrm{~min}$. The membrane was blocked with $5 \%$ milk in TBST ( $20 \mathrm{mM}$ Tris $\mathrm{pH} 7.6,150 \mathrm{mM} \mathrm{NaCl}$, $0.05 \%$ Tween-20) for $40 \mathrm{~min}$ at $37^{\circ} \mathrm{C}$ and for $20 \mathrm{~min}$ at room temperature. The primary antibodies, anti Mfa5-vWF or anti Mfa3 (Agrisera AB, Sweden), were diluted 1:20,000 in 3\% milk in TBST and incubated with the membrane on a shaker for 60 min. After washing for 10 min three times with TBST, the secondary antibody (abcam, Sweden) was applied at a 1:50,000 dilution in 3\% milk in TBST for 60 min. After washing three times with TBST, the bound secondary antibody was detected by chemiluminescence using BCIP/NBT (Promega).

\section{Crystallization, data collection, and structure determination}

Initial screening was performed using Mfa521-1044 (longest construct) at $12 \mathrm{mg} \cdot \mathrm{mL}^{-1}$ and Mfa5138435 (predicted vWF domain) at $18 \mathrm{mg} \cdot \mathrm{mL}^{-1}$. Crystallization was performed by sitting-drop vapordiffusion at $18^{\circ} \mathrm{C}$ using commercially available screens from Molecular Dimensions and Hampton Research with a Mosquito robot (TTP Labtech). Mfa521-1044 crystals grew in 12-17\% PEG 3350, 80 $\mathrm{mM} \mathrm{Na} 2 \mathrm{PO} 4 \mathrm{pH}$ 6, $3 \mathrm{mM} \mathrm{CaCl} 2$, and $3 \mathrm{mM} \mathrm{MgCl} 2$ and were cryoprotected in mother liquor supplemented with $25 \%$ glycerol and flash-cooled in liquid nitrogen. A native dataset was collected at beamline MX14.1 at the Berlin Electron Storage Ring Society for Synchrotron Radiation (Germany). One single crystal of Mfa5138-435, grown in 20\% PEG 500, 10\% PEG 20000, 0.1 M Sodium HEPES/MOPS pH 7.5, and $0.1 \mathrm{M}$ Glu/Ala/Gly/Lys/Ser-mix was obtained. The crystal was directly flash-cooled without additional cryoprotectants. A high redundancy sulfur single-wavelength anomalous dispersion (SAD) dataset was collected at beamline ID29 at the European Synchrotron Radiation Facility (Grenoble, France) [59]. All datasets were processed with XDS [60], and the sulfur SAD data were combined with BLEND [61]. Phase determination, refinement, and automated model building were performed in the PHENIX suite [62]. The initial solution of Mfa5138-435 was used as a molecular replacement model for Mfa521-1044, which was built by BUCCANEER [63] and with cycles of manual building in COOT [64] followed by refinement with PHENIX-refine. The final structures were validated using MOLPROBITY [65] and PDBSUM [66], and analyzed with CATH [34], SALIGN [67], and DALI [37]. Figures were prepared with PyMOL [68].

\section{Thermal shift assay}

A stable construct representing the long form of the Mfa5 structure, Mfa599-664 and its isopeptide mutant Mfa599-664 (K111A), were analyzed for thermal stability. The protein samples at $1 \mathrm{mg} \cdot \mathrm{mL}^{-1}$ were mixed with $20 \mathrm{mM}$ Tris buffer $\mathrm{pH} 8.5$ and 50x SYPRO Orange (Molecular Probes). The assay was conducted using a stepwise temperature increase from $10^{\circ} \mathrm{C}$ to $95^{\circ} \mathrm{C}$ in a CFX Connect instrument (BioRad) at a rate of $2^{\circ} \mathrm{C} / \mathrm{min}$. Data points were collected with the FAM channel (excitation $450-490$ 
$\mathrm{nm} /$ detection $510-530 \mathrm{~nm}$ ) after each $0.5^{\circ} \mathrm{C}$ with $3 \mathrm{~s}$ equilibration time. Plotting the first derivative of the measured fluorescence at each data point versus the temperature in Microsoft Excel allowed the calculation of the inflection point, and the minima were referred to as the melting temperatures (Tms). Each point was measured in triplicate, and their average value was used.

\section{Mass spectrometry}

The accurate molecular mass for Mfa599-664 and its K111A mutant was determined by electrospray ionization time of flight (ESI-TOF) mass spectrometry. Purified protein, $4 \mu \mathrm{L}$ at $10 \mathrm{mg} \cdot \mathrm{mL}^{-1}$, was separated on a C8 column using a gradient of $0.1 \%$ aqueous formic acid to $0.1 \%$ formic acid in acetonitrile over 3 min on an Agilent 1200 HPLC system. The sample was subsequently ionized in an acquisition mode operated at $4 \mathrm{GHz}$ on an Agilent $6230 \mathrm{LC}-\mathrm{TOF} / \mathrm{MS}$. The acquired total ion chromatogram was deconvoluted in the MassHunter Qualitative Analysis software (Agilent; V: B.07.00).

\section{Transmission electron microscopy}

Purified $P$. gingivalis fimbriae $(3 \mu \mathrm{L})$ were applied to a carbon coated and glow-discharged copper grid (CF300-Cu, Electron Microscopy Sciences) and incubated for $3 \mathrm{~min}$. The excess sample was removed by blotting, and the grid was washed with two drops of water and stained with $1.5 \% \mathrm{w} / \mathrm{v}$ uranyl acetate for $15 \mathrm{~s}$ two times. A Ceta CMOS camera ( $4 \mathrm{k} \times 4 \mathrm{k}$ pixels, $\mathrm{FEI}$ ) connected to a Talos $\mathrm{L} 120 \mathrm{C}$ transmission electron microscope (FEl, Umeå Core Facility for Electron Microscopy, Sweden) operating at $120 \mathrm{kV}$ was used to examine the negative-stained samples. Images were recorded using the TIA software (FEI).

\section{Visualization}

Sequence alignments calculated in MULTALIGN [69] were redrawn in ESPRIPT [70] for better visibility.

\section{References}

1. Fronzes $R$, Remaut $H$, Waksman $G$. Architectures and biogenesis of non-flagellar protein appendages in Gram-negative bacteria. EMBO J. 2008;27(17):2271-80. Epub 2008/08/01. doi: 10.1038/emboj.2008.155. PubMed PMID: 18668121; PubMed Central PMCID:

2. Hospenthal MK, Zyla D, Costa TRD, Redzej A, Giese C, Lillington J, et al. The Cryoelectron Microscopy Structure of the Type 1 Chaperone-Usher Pilus Rod. Structure. 2017;25(12):1829-38 e4. Epub 2017/11/14. doi: 10.1016/j.str.2017.10.004. PubMed PMID: 29129382; PubMed Central PMCID:

3. Krishnan V. Pilins in gram-positive bacteria: A structural perspective. IUBMB Life. 2015;67(7):533-43. Epub 2015/07/17. doi: 10.1002/iub.1400. PubMed PMID:

4. Lukaszczyk M, Pradhan B, Remaut $H$. The Biosynthesis and Structures of Bacterial Subcell Biochem. 2019;92:369-413. Epub 2019/06/20. doi: 10.1007/978-3-030-18768-2_12. PubMed PMID: 31214993.

5. Mandlik A, Das A, Ton-That H. The molecular switch that activates the cell wall anchoring step of pilus assembly in gram-positive bacteria. Proc Natl Acad Sci U S A. 2008;105(37):14147-52. Epub 
2008/09/10. doi: 10.1073/pnas.0806350105. PubMed PMID: 18779588; PubMed Central PMCID:

6. Kang HJ, Coulibaly F, Clow F, Proft T, Baker EN. Stabilizing isopeptide bonds revealed in grampositive bacterial pilus structure. Science. 2007;318(5856):1625-8. Epub 2007/12/08. doi:

10.1126/science.1145806. PubMed PMID:

7. Ton-That $\mathrm{H}$, Schneewind $\mathrm{O}$. Assembly of pili on the surface of Corynebacterium diphtheriae. Mol Microbiol. 2003;50(4):1429-38. Epub 2003/11/19. doi: 10.1046/j.1365- 2958.2003.03782.x. PubMed PMID:

8. Darveau RP, Hajishengallis G, Curtis MA. Porphyromonas gingivalis as a potential community activist for disease. J Dent Res. 2012;91(9):816-20. Epub 2012/07/10. doi: 10.1177/0022034512453589. PubMed PMID: 22772362; PubMed Central PMCID: PMCPMC3420389.

9. Park Y, Simionato MR, Sekiya K, Murakami Y, James D, Chen W, et al. Short fimbriae of Porphyromonas gingivalis and their role in coadhesion with Streptococcus gordonii. Infect Immun. 2005;73(7):3983-9. Epub 2005/06/24. doi: 10.1128/IAI.73.7.3983-3989.2005. PubMed PMID: 15972485; PubMed Central PMCID:

10. Wright CJ, Wu H, Melander RJ, Melander C, Lamont RJ. Disruption of heterotypic community development by Porphyromonas gingivalis with small molecule inhibitors. Mol Oral Microbiol. 2014;29(5):185-93. Epub 2014/06/06. doi: 10.1111/omi.12060. PubMed PMID: 24899524; PubMed Central PMCID:

11. D'Aiuto F, Parkar M, Andreou G, Suvan J, Brett PM, Ready D, et al. Periodontitis and systemic inflammation: control of the local infection is associated with a reduction in serum inflammatory markers. J Dent Res. 2004;83(2):156-60. Epub 2004/01/27. doi: 10.1177/154405910408300214. PubMed PMID:

12. Noack B, Genco RJ, Trevisan M, Grossi S, Zambon JJ, De Nardin E. Periodontal infections contribute to elevated systemic C-reactive protein level. J Periodontol. 2001;72(9):1221-7. Epub 2001/10/02. doi: 10.1902/jop.2000.72.9.1221. PubMed PMID: 11577954.

13. Pejcic A, Kesic LJ, Milasin J. C-reactive protein as a systemic marker of inflammation in periodontitis. Eur J Clin Microbiol Infect Dis. 2011;30(3):407-14. Epub 2010/11/09. doi: 10.1007/s10096-0101101-1. PubMed PMID:

14. Demmer RT, Desvarieux M. Periodontal infections and cardiovascular disease: the heart of the matter. J Am Dent Assoc. 2006;137 Suppl:14S-20S; quiz 38S. Epub 2006/10/03. doi: 10.14219/jada.archive.2006.0402. PubMed PMID:

15. Gogeneni H, Buduneli N, Ceyhan-Ozturk B, Gumus P, Akcali A, Zeller I, et al. Increased infection with key periodontal pathogens during gestational diabetes mellitus. J Clin Periodontol. 2015;42(6):50612. Epub 2015/05/12. doi: 10.1111/jcpe.12418. PubMed PMID: 25959628; PubMed Central PMCID:

16. Kebschull M, Demmer RT, Papapanou PN. "Gum bug, leave my heart alone!"- epidemiologic and mechanistic evidence linking periodontal infections and atherosclerosis. J Dent Res. 2010;89(9):879902. Epub 2010/07/20. doi: 10.1177/0022034510375281. PubMed PMID: 20639510; PubMed Central PMCID: 
17. Madianos PN, Bobetsis YA, Offenbacher S. Adverse pregnancy outcomes (APOs) and periodontal disease: pathogenic mechanisms. J Periodontol. 2013;84(4 Suppl):S170-80. Epub 2013/05/03. doi: 10.1902/jop.2013.1340015. PubMed PMID:

18. Maresz KJ, Hellvard A, Sroka A, Adamowicz K, Bielecka E, Koziel J, et al. Porphyromonas gingivalis facilitates the development and progression of destructive arthritis through its unique bacterial peptidylarginine deiminase (PAD). PLoS Pathog. 2013;9(9):e1003627. Epub 2013/09/27. doi: 10.1371/journal.ppat.1003627. PubMed PMID: 24068934; PubMed Central PMCID:

19. Ye L, Jiang Y, Liu W, Tao H. Correlation between periodontal disease and oral cancer risk: A metaanalysis. J Cancer Res Ther. 2016;12(Supplement):C237-C40. Epub 2017/02/24. doi: 10.4103/09731482.200746. PubMed PMID:

20. Dominy SS, Lynch C, Ermini F, Benedyk M, Marczyk A, Konradi A, et al. Porphyromonas gingivalis in Alzheimer's disease brains: Evidence for disease causation and treatment with small-molecule inhibitors. Sci Adv. 2019;5(1):eaau3333. Epub 2019/02/13. doi: 10.1126/sciadv.aau3333. PubMed PMID: 30746447; PubMed Central PMCID: PMCPMC6357742.

21. Lamont RJ, Bevan CA, Gil S, Persson RE, Rosan B. Involvement of Porphyromonas gingivalis fimbriae in adherence to Streptococcus gordonii. Oral Microbiol Immunol. 1993;8(5):272-6. Epub 1993/10/01. doi: 10.1111/j.1399-302x.1993.tb00573.x. PubMed PMID:

22. Nakagawa I, Inaba H, Yamamura T, Kato T, Kawai S, Ooshima T, et al. Invasion of epithelial cells and proteolysis of cellular focal adhesion components by distinct types of Porphyromonas gingivalis fimbriae. Infect Immun. 2006;74(7):3773-82. Epub 2006/06/23. doi: 10.1128/IAI.01902-05. PubMed PMID: 16790749; PubMed Central PMCID: PMCPMC1489697.

23. Hajishengallis G. Periodontitis: from microbial immune subversion to systemic inflammation. Nat Rev Immunol. 2015;15(1):30-44. Epub 2014/12/24. doi: 1038/nri3785. PubMed PMID: 25534621; PubMed Central PMCID: PMCPMC4276050.

24. Sojar HT, Sharma A, Genco RJ. Porphyromonas gingivalis fimbriae bind to cytokeratin of epithelial cells. Infect Immun. 2002;70(1):96-101. Epub 2001/12/19. doi: 10.1128/iai.70.1.96-101.2002. PubMed PMID: 11748168; PubMed Central PMCID: PMCPMC127629.

25. Zhang W, Ju J, Rigney T, Tribble GD. Fimbriae of Porphyromonas gingivalis are important for initial invasion of osteoblasts, but not for inhibition of their differentiation and mineralization. $J$ Periodontol. 2011;82(6):909-16. Epub 2010/12/30. doi: 10.1902/jop.2010.100501. PubMed PMID: 21189086.

26. Hamada N, Sojar HT, Cho MI, Genco RJ. Isolation and characterization of a minor fimbria from Porphyromonas gingivalis. Infect Immun. 1996;64(11):4788-94. Epub 1996/11/01. PubMed PMID: 8890240; PubMed Central PMCID:

27. Yoshimura F, Takahashi K, Nodasaka Y, Suzuki T. Purification and characterization of a novel type of fimbriae from the oral anaerobe Bacteroides gingivalis. J Bacteriol. 1984;160(3):949-57. Epub 1984/12/01. PubMed PMID: 6150029; PubMed Central PMCID: PMCPMC215801. 
28. Enersen M, Nakano K, Amano A. Porphyromonas gingivalis fimbriae. J Oral Microbiol. 2013;5. Epub 2013/05/15. doi: 10.3402/jom.v5i0.20265. PubMed PMID: 23667717; PubMed Central PMCID:

29. Yoshimura F, Murakami Y, Nishikawa K, Hasegawa Y, Kawaminami S. Surface components of Porphyromonas gingivalis. J Periodontal Res. 2009;44(1):1-12. Epub 2008/11/01. doi: 10.1111/j.1600-0765.2008.01135.x. PubMed PMID:

30. Shoji M, Yoshimura A, Yoshioka H, Takade A, Takuma Y, Yukitake H, et al. Recombinant Porphyromonas gingivalis FimA preproprotein expressed in Escherichia coli is lipidated and the mature or processed recombinant FimA protein forms a short filament in vitro. Can J Microbiol. 2010;56(11):959-67. Epub 2010/11/16. doi: 10.1139/w10-084. PubMed PMID:

31. Okuda S, Tokuda H. Lipoprotein sorting in bacteria. Annu Rev Microbiol. 2011;65:239- 59. Epub 2011/06/15. doi: 10.1146/annurev-micro-090110-102859. PubMed PMID: 21663440.

32. Lasica AM, Ksiazek M, Madej M, Potempa J. The Type IX Secretion System (T9SS): Highlights and Recent Insights into Its Structure and Function. Front Cell Infect Microbiol. 2017;7:215. Epub 2017/06/13. doi: 10.3389/fcimb.2017.00215. PubMed PMID: 28603700; PubMed Central PMCID:

33. Hasegawa Y, lijima Y, Persson K, Nagano K, Yoshida Y, Lamont RJ, et al. Role of Mfa5 in Expression of Mfa1 Fimbriae in Porphyromonas gingivalis. J Dent Res. 2016;95(11):1291-7. Epub 2016/06/22. doi: 10.1177/0022034516655083. PubMed PMID: 27323953; PubMed Central PMCID:

34. Dawson NL, Lewis TE, Das S, Lees JG, Lee D, Ashford P, et al. CATH: an expanded resource to predict protein function through structure and sequence. Nucleic Acids 2017;45(D1):D289-D95. Epub 2016/12/03. doi: 10.1093/nar/gkw1098. PubMed PMID: 27899584; PubMed Central PMCID: PMCPMC5210570.

35. Vengadesan K, Narayana SV. Structural biology of Gram-positive bacterial adhesins. Protein Sci. 2011;20(5):759-72. Epub 2011/03/16. doi: 10.1002/pro.613. PubMed PMID: 21404359; PubMed Central PMCID:

36. Whittaker CA, Hynes RO. Distribution and evolution of von Willebrand/integrin A domains: widely dispersed domains with roles in cell adhesion and elsewhere. Mol Biol Cell. 2002;13(10):3369-87. Epub 2002/10/22. doi: 10.1091/mbc.e02-05-0259. PubMed PMID: 12388743; PubMed Central PMCID:

37. Holm L, Rosenstrom P. Dali server: conservation mapping in 3D. Nucleic Acids Res. 2010;38(Web Server issue):W545-9. Epub 2010/05/12. doi: 10.1093/nar/gkq366. PubMed PMID: 20457744; PubMed Central PMCID:

38. Izore T, Contreras-Martel C, El Mortaji L, Manzano C, Terrasse R, Vernet T, et al. Structural basis of host cell recognition by the pilus adhesin from Streptococcus pneumoniae. Structure. 2010;18(1):106-15. Epub 2010/02/16. doi: 10.1016/j.str.2009.10.019. PubMed PMID: 20152157.

39. Krishnan V, Dwivedi P, Kim BJ, Samal A, Macon K, Ma X, et al. Structure of Streptococcus agalactiae tip pilin GBS104: a model for GBS pili assembly and host interactions. Acta Crystallogr D Biol Crystallogr. 2013;69(Pt 6):1073-89. Epub 2013/05/23. doi: 10.1107/S0907444913004642. PubMed PMID: 23695252; PubMed Central PMCID: PMCPMC3663123. 
40. Emsley J, Knight CG, Farndale RW, Barnes MJ, Liddington RC. Structural basis of collagen recognition by integrin alpha2beta1. Cell. 2000;101(1):47-56. Epub 2000/04/25. doi: 10.1016/S00928674(00)80622-4. PubMed PMID:

41. Van Agthoven JF, Xiong JP, Alonso JL, Rui X, Adair BD, Goodman SL, et al. Structural basis for pure antagonism of integrin alphaVbeta3 by a high-affinity form of fibronectin. Nat Struct Mol Biol. 2014;21(4):383-8. Epub 2014/03/25. doi: 10.1038/nsmb.2797. PubMed PMID: 24658351; PubMed Central PMCID:

42. Hasegawa Y, Iwami J, Sato K, Park Y, Nishikawa K, Atsumi T, et al. Anchoring and length regulation of Porphyromonas gingivalis Mfa1 fimbriae by the downstream gene product Mfa2. Microbiology. 2009;155(Pt 10):3333-47. Epub 2009/07/11. doi: 10.1099/mic.0.028928-0. PubMed PMID: 19589838; PubMed Central PMCID: PMCPMC2810400.

43. Ruer S, Pinotsis N, Steadman D, Waksman G, Remaut H. Virulence-targeted Antibacterials: Concept, Promise, and Susceptibility to Resistance Mechanisms. Chem Biol Drug Des. 2015;86(4):379-99. Epub 2015/01/16. doi: 10.1111/cbdd.12517. PubMed PMID: 25589217.

44. Xu Q, Shoji M, Shibata S, Naito M, Sato K, Elsliger MA, et al. A Distinct Type of Pilus from the Human Microbiome. Cell. 2016;165(3):690-703. Epub 2016/04/12. doi: 10.1016/j.cell.2016.03.016. PubMed PMID: 27062925; PubMed Central PMCID: PMCPMC4842110.

45. Kloppsteck P, Hall M, Hasegawa Y, Persson K. Structure of the fimbrial protein Mfa4 from Porphyromonas gingivalis in its precursor form: implications for a donor-strand complementation mechanism. Sci Rep. 2016;6:22945. Epub 2016/03/15. doi: 10.1038/srep22945. PubMed PMID: 26972441; PubMed Central PMCID:

46. Hall M, Hasegawa Y, Yoshimura F, Persson K. Structural and functional characterization of shaft, anchor, and tip proteins of the Mfa1 fimbria from the periodontal pathogen Porphyromonas gingivalis. Sci Rep. 2018;8(1):1793. Epub 2018/01/31. doi: 10.1038/s41598-018-20067-z. PubMed PMID: 29379120; PubMed Central PMCID: PMCPMC5789003.

47. Lee JY, Miller DP, Wu L, Casella CR, Hasegawa Y, Lamont RJ. Maturation of the Mfa1 Fimbriae in the Oral Pathogen Porphyromonas gingivalis. Front Cell Infect Microbiol. 2018;8:137. Epub 2018/06/06. doi: 10.3389/fcimb.2018.00137. PubMed PMID: 29868494; PubMed Central PMCID:

48. Roberts AP, Kreth J. The impact of horizontal gene transfer on the adaptive ability of the human oral microbiome. Front Cell Infect Microbiol. 2014;4:124. Epub 2014/09/25. doi:

10.3389/fcimb.2014.00124. PubMed PMID: 25250243; PubMed Central PMCID: PMCPMC4157583.

49. Almagro Armenteros JJ, Tsirigos KD, Sonderby CK, Petersen TN, Winther O, Brunak S, et al. SignalP 5.0 improves signal peptide predictions using deep neural networks. Nat Biotechnol. 2019;37(4):4203. Epub 2019/02/20. doi: 10.1038/s41587-019-0036-z. PubMed PMID:

50. Sigrist CJ, de Castro E, Cerutti L, Cuche BA, Hulo N, Bridge A, et al. New and continuing developments at PROSITE. Nucleic Acids Res. 2013;41(Database issue):D344-7. Epub 2012/11/20. doi: 10.1093/nar/gks1067. PubMed PMID: 23161676; PubMed Central PMCID: 
51. Mitchell AL, Attwood TK, Babbitt PC, Blum M, Bork P, Bridge A, et al. InterPro in 2019: improving coverage, classification and access to protein sequence annotations. Nucleic Acids Res. 2019;47(D1):D351-D60. Epub 2018/11/07. doi: 10.1093/nar/gky1100. PubMed PMID: 30398656; PubMed Central PMCID:

52. Marchler-Bauer A, Bo Y, Han L, He J, Lanczycki CJ, Lu S, et al. CDD/SPARCLE: functional classification of proteins via subfamily domain architectures. Nucleic Acids 2017;45(D1):D200-D3. Epub 2016/12/03. doi: 10.1093/nar/gkw1129. PubMed PMID: 27899674; PubMed Central PMCID: PMCPMC5210587.

53. Garnier J, Gibrat JF, Robson B. GOR method for predicting protein secondary structure from amino acid sequence. Methods Enzymol. 1996;266:540-53. Epub 1996/01/01. doi: 10.1016/s00766879(96)66034-0. PubMed PMID:

54. Cheng J, Randall AZ, Sweredoski MJ, Baldi P. SCRATCH: a protein structure and structural feature prediction server. Nucleic Acids Res. 2005;33(Web Server issue):W72-6. Epub 2005/06/28. doi: 10.1093/nar/gki396. PubMed PMID: 15980571; PubMed Central PMCID:

55. Buchan DW, Minneci F, Nugent TC, Bryson K, Jones Scalable web services for the PSIPRED Protein Analysis Workbench. Nucleic Acids Res. 2013;41(Web Server issue):W349-

56. Epub 2013/06/12. doi: 10.1093/nar/gkt381. PubMed PMID: 23748958; PubMed Central PMCID: PMCPMC3692098.

57. Kelley LA, Mezulis S, Yates CM, Wass MN, Sternberg MJ. The Phyre2 web portal for protein modeling, prediction and analysis. Nat Protoc. 2015;10(6):845-58. Epub 2015/05/08. doi: 10.1038/nprot.2015.053. PubMed PMID: 25950237; PubMed Central PMCID: PMCPMC5298202.

58. Klock HE, Koesema EJ, Knuth MW, Lesley SA. Combining the polymerase incomplete primer extension method for cloning and mutagenesis with microscreening to accelerate structural genomics efforts. Proteins. 2008;71(2):982-94. Epub 2007/11/16. doi: 10.1002/prot.21786. PubMed PMID:

59. Bogomolovas J, Simon B, Sattler M, Stier G. Screening of fusion partners for high yield expression and purification of bioactive viscotoxins. Protein Expr Purif. 2009;64(1):16-23. Epub 2008/11/06. doi: 10.1016/j.pep.2008.10.003. PubMed PMID:

60. de Sanctis D, Beteva A, Caserotto H, Dobias F, Gabadinho J, Giraud T, et al. ID29: a high-intensity highly automated ESRF beamline for macromolecular crystallography experiments exploiting anomalous scattering. J Synchrotron Radiat. 2012;19(Pt 3):455-61. Epub 2012/04/20. doi: 10.1107/S0909049512009715. PubMed PMID:

61. Kabsch W. Xds. Acta Crystallogr D Biol Crystallogr. 2010;66(Pt 2):125-32. Epub 2010/02/04. doi: 10.1107/S0907444909047337. PubMed PMID: 20124692; PubMed Central PMCID:

62. Foadi J, Aller P, Alguel Y, Cameron A, Axford D, Owen RL, et al. Clustering procedures for the optimal selection of data sets from multiple crystals in macromolecular crystallography. Acta Crystallogr D Biol Crystallogr. 2013;69(Pt 8):1617-32. Epub 2013/07/31. doi: 10.1107/S0907444913012274. PubMed PMID: 23897484; PubMed Central PMCID: PMCPMC3727331. 
63. Adams PD, Afonine PV, Bunkoczi G, Chen VB, Davis IW, Echols N, et al. PHENIX: a comprehensive Python-based system for macromolecular structure solution. Acta Crystallogr D Biol Crystallogr. 2010;66(Pt 2):213-21. Epub 2010/02/04. doi: 10.1107/S0907444909052925. PubMed PMID: 20124702; PubMed Central PMCID: PMCPMC2815670.

64. Cowtan K. The Buccaneer software for automated model building. 1. Tracing protein chains. Acta Crystallogr D Biol Crystallogr. 2006;62(Pt 9):1002-11. Epub 2006/08/25. doi: 10.1107/S0907444906022116. PubMed PMID:

65. Emsley P, Lohkamp B, Scott WG, Cowtan K. Features and development of Coot. Acta Crystallogr D Biol Crystallogr. 2010;66(Pt 4):486-501. Epub 2010/04/13. doi: 10.1107/S0907444910007493. PubMed PMID: 20383002; PubMed Central PMCID: PMCPMC2852313.

66. Chen VB, Arendall WB, 3rd, Headd JJ, Keedy DA, Immormino RM, Kapral GJ, et al. MolProbity: allatom structure validation for macromolecular crystallography. Acta Crystallogr D Biol Crystallogr. 2010;66(Pt 1):12-21. Epub 2010/01/09. doi: 10.1107/S0907444909042073. PubMed PMID: 20057044; PubMed Central PMCID: PMCPMC2803126.

67. Laskowski RA. PDBsum new things. Nucleic Acids Res. 2009;37(Database issue):D355-

68. Epub 2008/11/11. doi: 10.1093/nar/gkn860. PubMed PMID: 18996896; PubMed Central PMCID: PMCPMC2686501.

69. Braberg H, Webb BM, Tjioe E, Pieper U, Sali A, Madhusudhan MS. SALIGN: a web server for alignment of multiple protein sequences and structures. Bioinformatics. 2012;28(15):2072-3. Epub 2012/05/24. doi: 10.1093/bioinformatics/bts302. PubMed PMID: 22618536; PubMed Central PMCID:

70. Schrodinger, LLC. The PyMOL Molecular Graphics System, Version 1.8.

71. Corpet F. Multiple sequence alignment with hierarchical clustering. Nucleic Acids 1988;16(22):1088190. Epub 1988/11/25. doi: 10.1093/nar/16.22.10881. PubMed PMID: 2849754; PubMed Central PMCID: PMCPMC338945.

72. Robert $X$, Gouet P. Deciphering key features in protein structures with the new ENDscript server. Nucleic Acids Res. 2014;42(Web Server issue):W320-4. Epub 2014/04/23. doi: 10.1093/nar/gku316. PubMed PMID: 24753421; PubMed Central PMCID: PMCPMC4086106.

\section{Declarations}

\section{Acknowledgements}

This work was funded by Kempestiftelserna (SMK-1553 and SMK-1756.2) and the Swedish Research Council (KP, 2016-05009). Our thanks go to Dr. Linda Sandblad and Dr. Michael Hall (Umeå Core Facility for Electron Microscopy [UCEM], Sweden) for advice on sample preparation and data acquisition and to Prof. Yoshiaki Hasegawa for the P. gingivalis strains. We thank Mikael Lindberg at the Protein expertise platform, Umeå University for help with the initial cloning. 
We thank the beamline scientists at beamlines ID29 (European Synchrotron Radiadion Facility, Grenoble) and MX14.1 (Berliner Elektronenspeicherring-Gesellschaft für Synchrotronstrahlung, Berlin) for their support.

\section{Contributions}

T.V.H. performed all protein purifications, crystallizations, crystallography and electron microscopy. R. C. performed the preparation of $P$. gingivalis. K. P. supervised the work.

T.V.H. and K. P. wrote the manuscript with contributions from R.C.

\section{Tables}

Table 1:

Data collection and refinement statistics.

Statistics for the highest-resolution shell are shown in parentheses. 


\begin{tabular}{|c|c|c|}
\hline & Mfa5D1 (aa138-435) & Mfa5D1-D3 (aa98-664) \\
\hline \multicolumn{3}{|l|}{ Data collection } \\
\hline Beamline & ID29 ESRF & MX14.1 BESSY \\
\hline Wavelength $[\AA ̊]$ & 1.5400 & 0.9756 \\
\hline Resolution range [^]] & $37.85-1.85(1.89-1.85)$ & $46.98-1.79(1.86-1.79)$ \\
\hline Space group & P 1211 & P 212121 \\
\hline Unit cell [a / b / c in Å] & 35.20 / 110.08 / 38.18 & 50.72 / $72.81 / 184.49$ \\
\hline [a / b / g in $\left.{ }^{\circ}\right]$ & 90 / $98.11 / 90.5$ & $90 / 90 / 90$ \\
\hline Total reflections & 4117009 (123254) & 838439 (68089) \\
\hline Unique reflections & 23968 (1123) & $64113(6117)$ \\
\hline Multiplicity & $171.8(109.8)$ & $13.1(11.1)$ \\
\hline - anomalous & $85.6(54.1)$ & \\
\hline Completeness [\%] & $97.6(75.9)$ & $99.45(95.94)$ \\
\hline - anomalous & $97.4(74.6)$ & \\
\hline Mean I/sigma(I) & $75.6(24.0)$ & $19.66(1.87)$ \\
\hline Wilson B-factor [Å2] & 9.49 & 23.69 \\
\hline R-merge [\%] & $0.087(0.369)$ & $0.097(1.126)$ \\
\hline R-meas [\%] & $0.087(0.373)$ & $0.101(1.180)$ \\
\hline R-pim & & $0.028(0.345)$ \\
\hline $\mathrm{CC} 1 / 2$ & $1.0(1.0)$ & $0.999(0.646)$ \\
\hline \multicolumn{3}{|l|}{$\underline{\text { Refinement }}$} \\
\hline \multicolumn{3}{|l|}{ Reflections used in: } \\
\hline - refinement & 23902 (1911) & $64104(6115)$ \\
\hline -R-free & 2010 (172) & $3206(306)$ \\
\hline R-work [\%] & $0.1333(0.1978)$ & $0.1612(0.2407)$ \\
\hline R-free [\%] & $0.1789(0.2277)$ & $0.1981(0.2711)$ \\
\hline \multicolumn{3}{|l|}{ Number of: } \\
\hline - non-hydrogen atoms & 2751 & 5084 \\
\hline - protein & 2347 & 4461 \\
\hline
\end{tabular}




\begin{tabular}{|c|c|c|}
\hline - ligands & 15 & 17 \\
\hline - Solvent molecules & 389 & 606 \\
\hline - Protein residues & 296 & 566 \\
\hline RMS (bonds) [Å] & 0.010 & 0.011 \\
\hline RMS (angles) [] & 1.07 & 0.99 \\
\hline $\begin{array}{l}\text { Ramachandran favored } \\
\text { (\%) }\end{array}$ & 96.94 & 97.34 \\
\hline $\begin{array}{l}\text { Ramachandran allowed } \\
\text { (\%) }\end{array}$ & 3.06 & 2.48 \\
\hline $\begin{array}{l}\text { Ramachandran outliers } \\
\text { (\%) }\end{array}$ & 0.00 & 0.18 \\
\hline Rotamer outliers (\%) & 0.00 & 0.00 \\
\hline Clashscore & 2.35 & 1.69 \\
\hline Average B-factor & 11.78 & 28.21 \\
\hline - protein & 9.92 & 26.76 \\
\hline - ligands & 30.19 & 38.49 \\
\hline - solvent & 22.29 & 38.57 \\
\hline VM [Å3/Da] & 2.24 & 2.76 \\
\hline Monomers / AU & 1 & 1 \\
\hline Solvent content [\%] & 45.00 & 55.39 \\
\hline PDBID & 6 tnj & 6 to 1 \\
\hline
\end{tabular}

Figures 


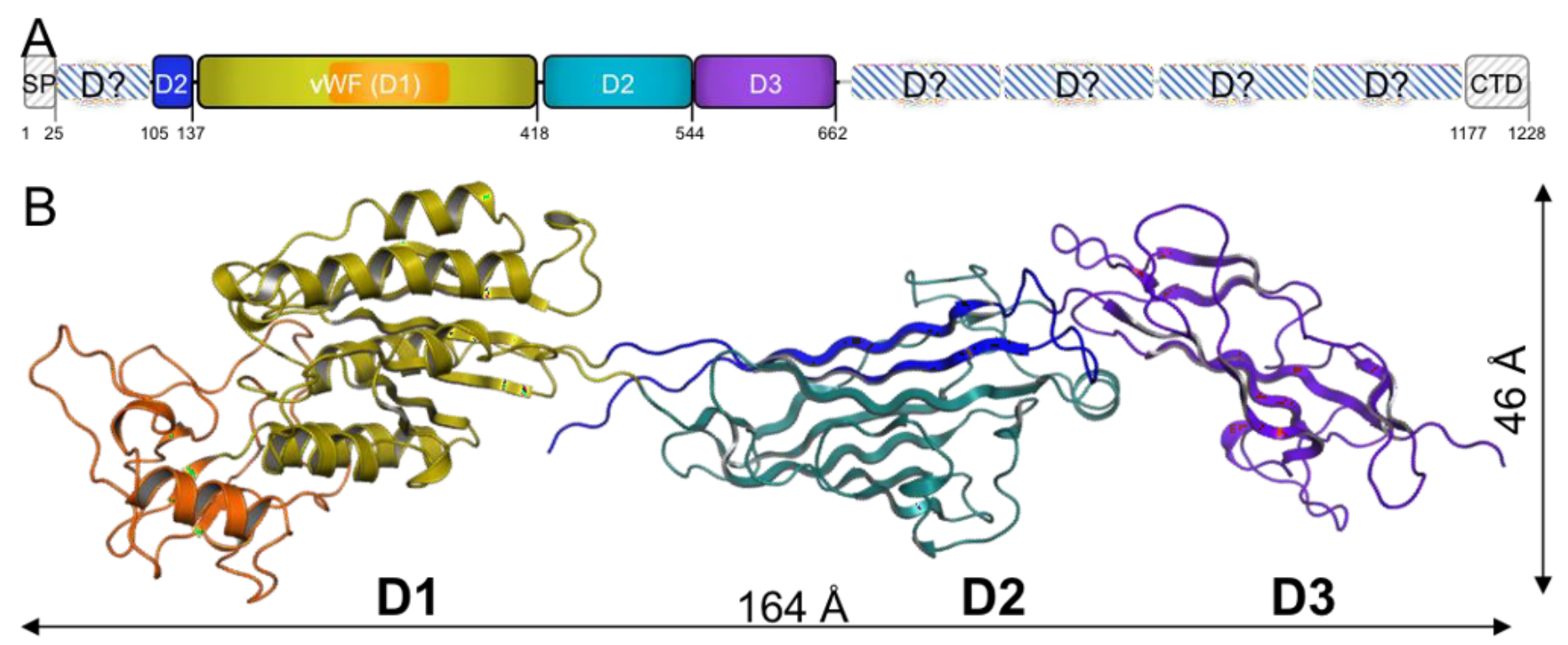

Figure 1

Domain arrangement and dimensions of Mfa5. A) a schematic arrangement of the domains of Mfa5 shown according to scale. The D1 domain is depicted in yellow (vWF-domain) and orange (ARM2 extension), the D2 domain is shown in blue and cyan, and the D3 domain is shown in purple. Potential missing IgG-like domains are shown in striped blue. The N-terminal signal peptide (SP) and the type-IX Cterminal signal domain (CTD) are depicted as grey boxes. B) Cartoon representation of the Mfa5 crystal structure, residues 99-664. The domains are colored as in A, and the domains are numbered and the dimensions are indicated. 


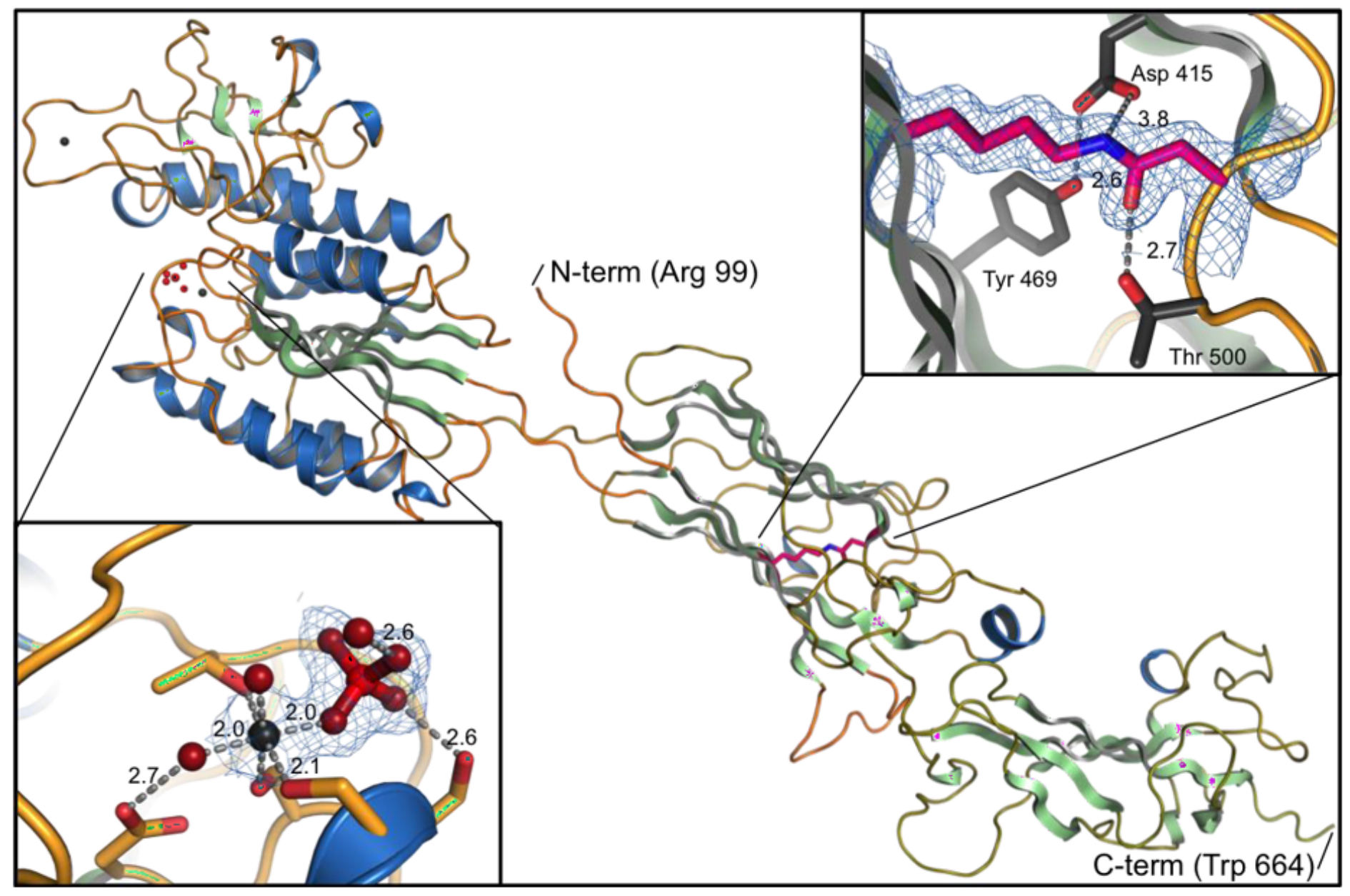

Figure 2

Structural features of Mfa5. Cartoon representation of Mfa5 showing the $\mathrm{N}$ - and C-termini, the two metal binding sites (black spheres), and the isopeptide bond (in magenta). Inserts on the top and bottom show the 2FoFc maps for the isopeptide bond and MIDAS motif at 1.2 sigma, respectively. Distances are shown as dashed lines with values in Ångström, the metal is shown as a black sphere, and the waters and the phosphate ion are shown in red. 
A
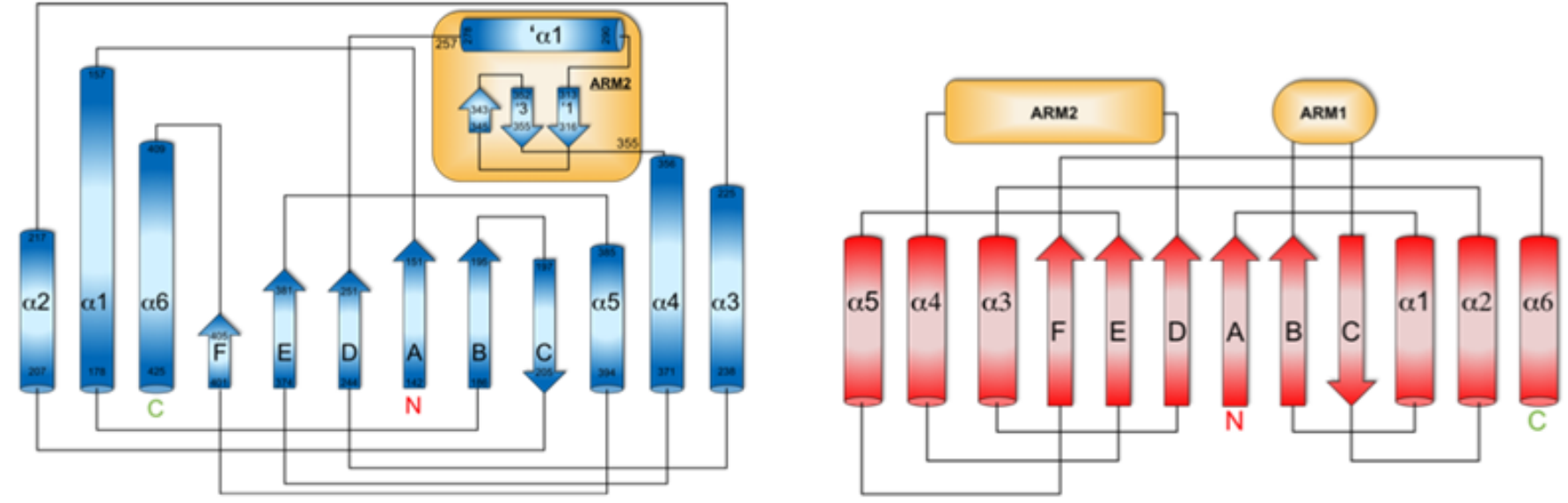

B
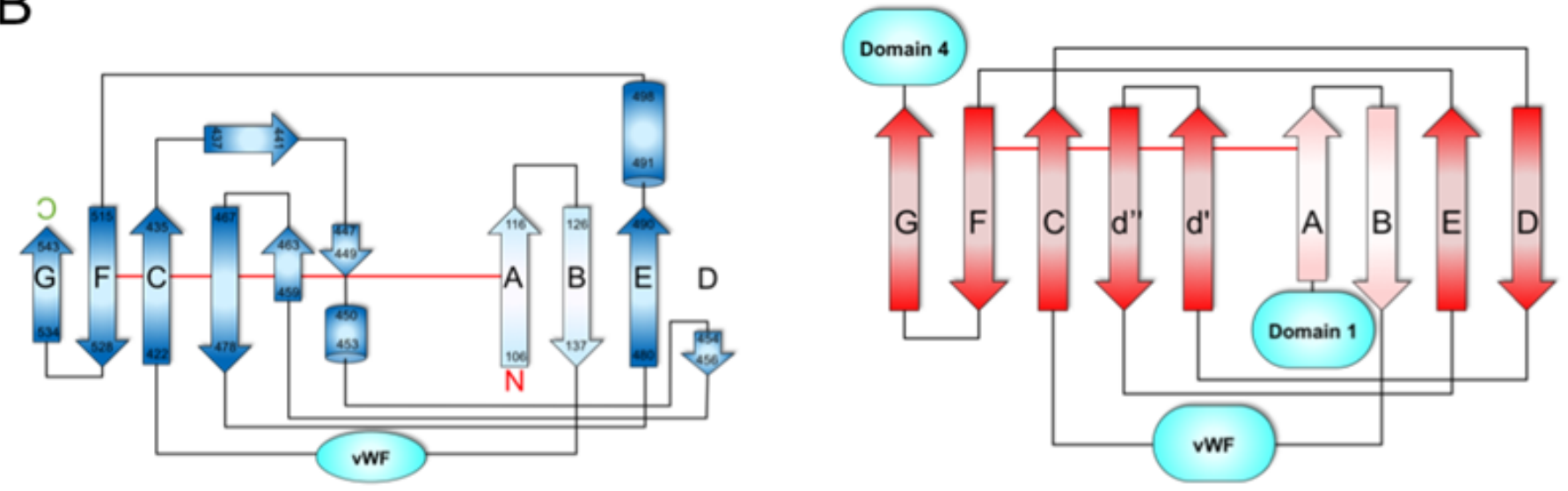

C
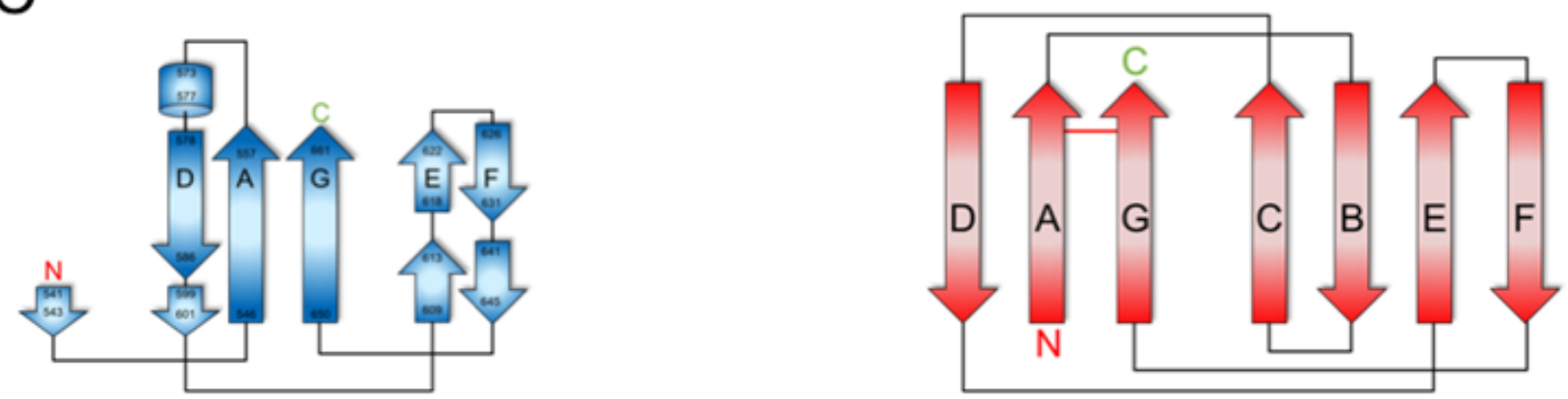

Figure 3

Comparison of the Mfa5 topology to Gram-positive fimbrial tip proteins. A) Mfa5 shares the overall fold of the Gram-positive vWF domains, as well as the position of the ARM2 region. B) Mfa5 D2 also shares the CnaA-like fold of GBS104 and RrgA and has the same domain structure. In addition, the positions of the two $\mathrm{N}$-terminal $\beta$-strands and the isopeptide bond are identical. C) In D3 the topology is similar to a CnaB-like domains, but $\beta$-strands $C$ and $B$ as well as the isopeptide bond are missing. Mfa5 is shown in 
blue and GBS104 in red. The isopeptide bonds are depicted as red lines. Mfa5 I shown to the left and the streptococcal protein to the right side of the figure.

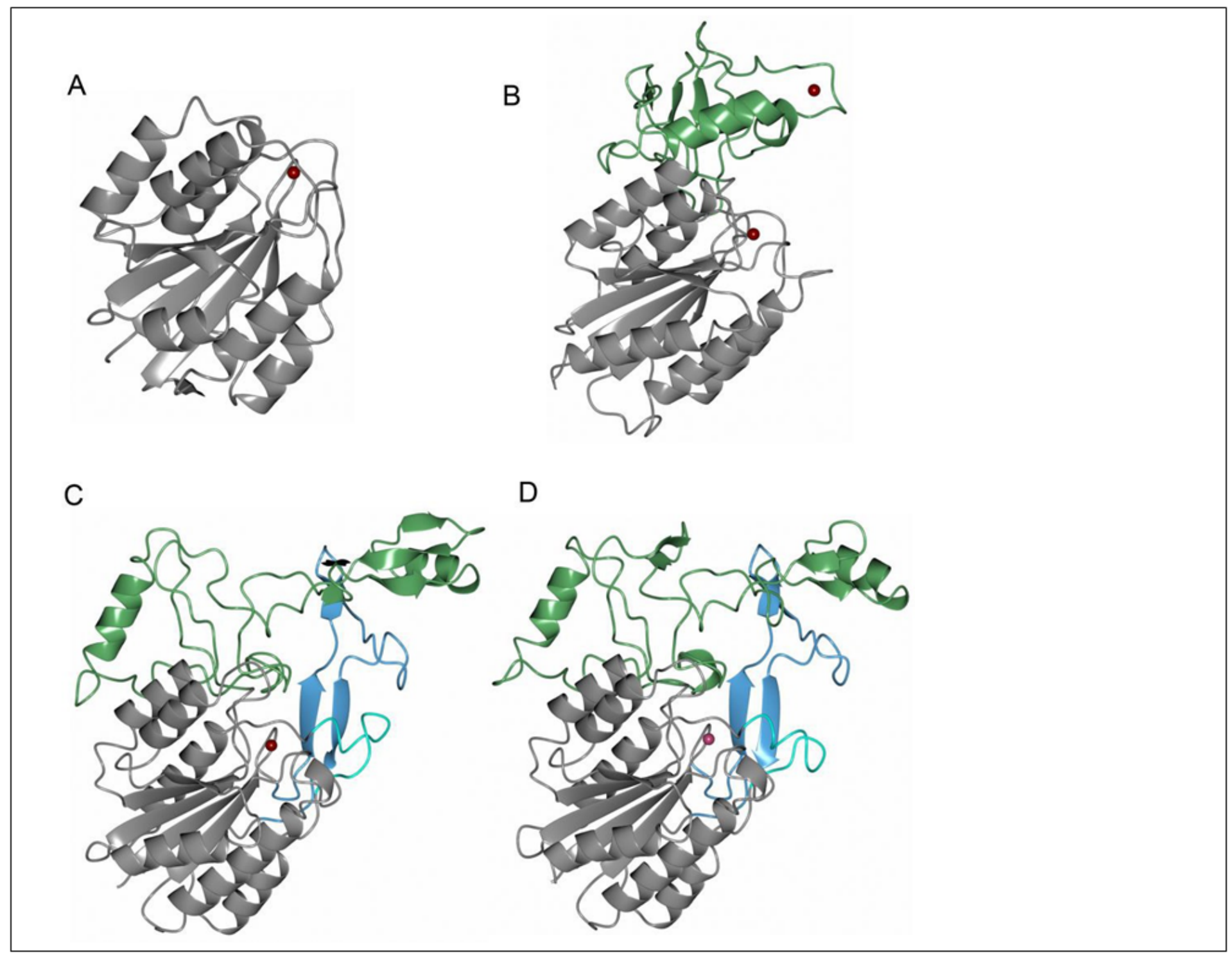

Figure 4

Comparison of the ARM subdomains of the VWF domains. Cartoon representation of the VWF domains of A) human integrin a2-I domain B) P. gingivalis Mfa5, C) streptococcal RrgA, and D) streptococcal GBS104. The vWF core is colored in grey. The ARM2 regions are colored in green, the ARM1 region in RrgA and GBS104 in blue and the loop located after a2 in RrgA and GBS104 in cyan. The metal ions are shown as dark red spheres. 


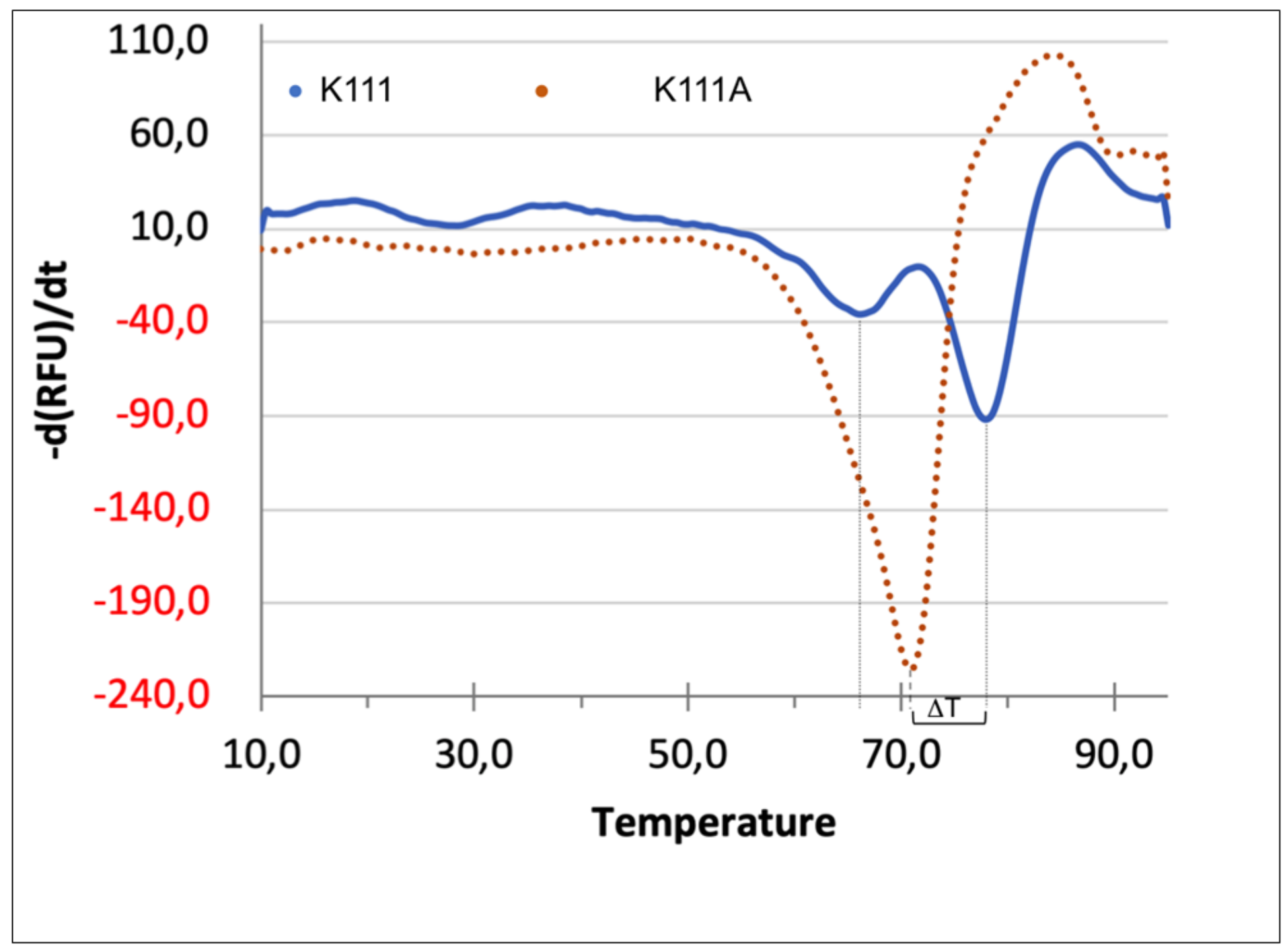

Figure 5

The isopeptide bond increases the stability of Mfa5. The first derivative of the measured fluorescence in a thermal shift assay of Mfa5(K111) (blue line) and Mfa5(K111A), (orange dotted line). The major unfolding peak is shifted 7 degrees when $\mathrm{K} 111$ and $\mathrm{K} 111 \mathrm{~A}$ are compared. 


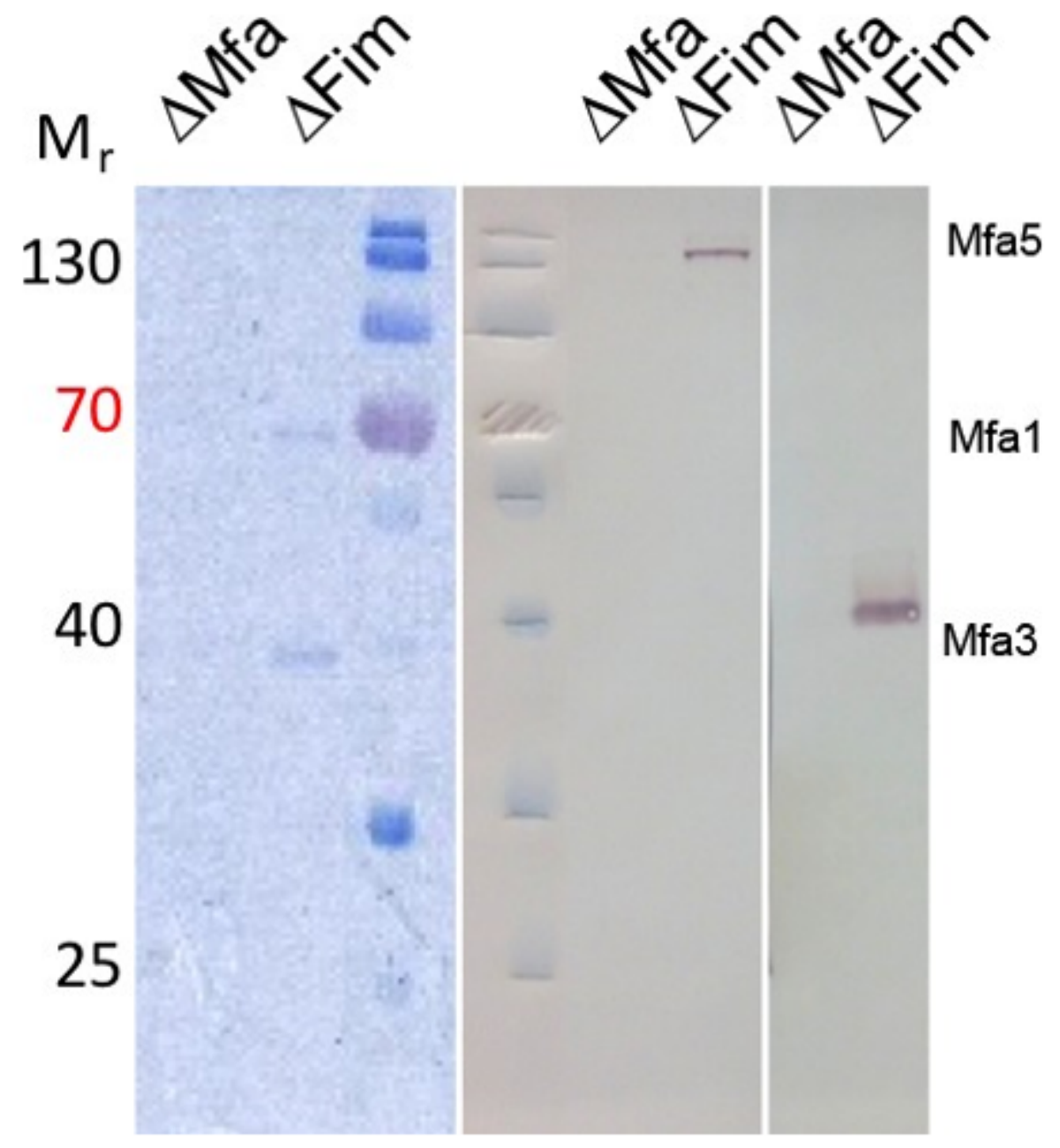

Figure 6

Validation of fimbrial proteins. Antibodies raised against the recombinant Mfa5 vWF domain and Mfa3 were used on native fimbrial purifications. The left panel shows Coomassie stained bands corresponding to Mfa3 and Mfa1. The middle and right panels show western blots of a fimbrial preparation from $\Delta \mathrm{mfa}$ and $\Delta$ fim mutants, respectively. The anti-Mfa5vWF antibody detects a high molecular weight protein, corresponding to Mfa5, in the $\Delta \mathrm{fim}$ mutant, and the anti-Mfa3 antibody detects a protein corresponding to Mfa3 in the $\Delta$ fim mutant. 


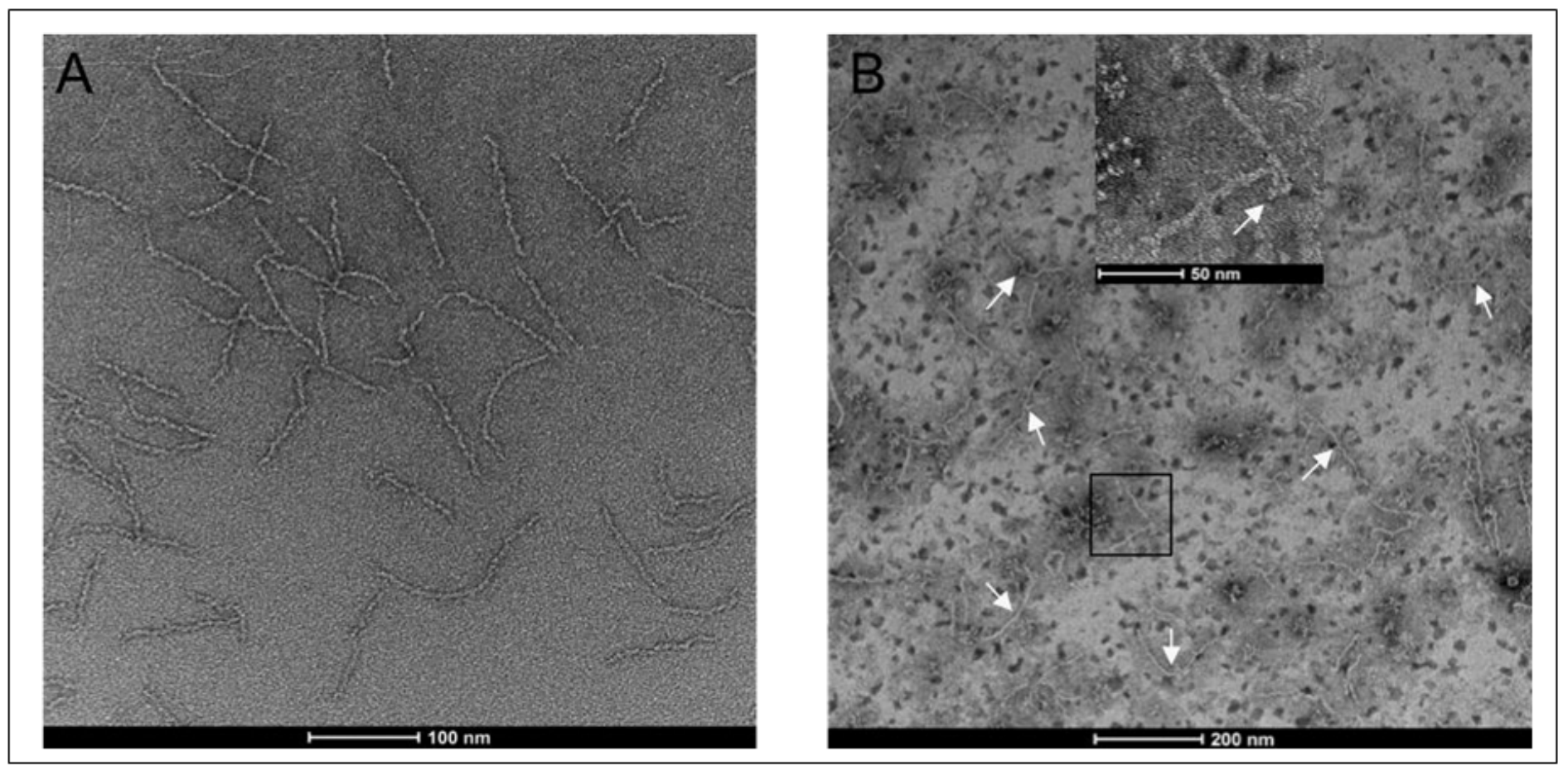

Figure 7

Mfa5 localization on the tip. Negative staining of the native purified Mfa-fimbriae A) and in complex with an Mfa5-vWF antibody B). The white arrows indicate the elongation and possible antibody connection. The insert in picture $B$ shows a magnification of the area in the black box.

\section{Supplementary Files}

This is a list of supplementary files associated with this preprint. Click to download.

- Mfa5supplementary.pdf 